\title{
Laparoscopic cholecystectomy in patients with a cardiovascular implantable electronic device: A single-center experience
}

\author{
Durmuş Ali Çetin, ${ }^{1}$ Hüseyin Çiyiltepe, ${ }^{2}$ Ebubekir Gündeş, ${ }^{3}$ Ulaş Aday, ${ }^{4}$ Emre Bozdağ, ${ }^{5}$ \\ Orhan Uzun, ${ }^{5}$ Kamuran Cumhur Değer, ${ }^{5}$ Mustafa Duman ${ }^{5}$ \\ 'Department of Gastroenterological Surgery, Şanlıurfa Training and Research Hospital, Şanlıurfa, Turkey \\ 2Department of Gastroenterological Surgery, Fatih Sultan Mehmet Training and Research Hospital, İstanbul, Turkey \\ ${ }^{3}$ Department of Gastroenterological Surgery, Diyarbakır Gazi Yaşargil Training and Research Hospital, Diyarbakır, Turkey \\ ${ }^{4}$ Department of Gastroenterological Surgery, Elazığ Training and Research Hospital, Elazığ, Turkey \\ ${ }^{5}$ Department of Gastroenterological Surgery, Kartal Koşuyolu High Speciality Training and Research Hospital, İstanbul, Turkey
}

\begin{abstract}
Introduction: The aim of this study was to investigate the feasibility of laparoscopic cholecystectomy (LC) with safe perioperative measures in patients with cardiac arrhythmia due to various heart diseases who had a cardiovascular implantable electronic device (CIED).

Materials and Methods: Cases of patients with a CIED and who underwent LC between January 2012 and December 2016 were retrospectively evaluated. The demographic data and the clinical, and perioperative results of the patients were analyzed.

Results: A total of 467 patients underwent LC at Kartal Koşuyolu Higher Specialty Training and Research Hospital's Gastroenterology Clinic between January 2012 and December 2016. Eight (0.017\%) patients had a CIED. One (12.5\%) of these patients was male, and $7(87.5 \%)$ were female. The mean age of the patients was 53.2 years (range: $28-82$ years). All of the patients were taken into surgery as a result of symptomatic cholelithiasis. Five patients had a dual-chamber rate-modulated pacing pacemaker, while 3 patients had an implantable cardioverter/defibrillator. No unexpected cardiac complications were seen in the perioperative period in any of the patients.

Conclusion: The results of this study suggest that LC can safely be performed in patients with a CIED at experienced centers with the appropriate perioperative management.
\end{abstract}

Keywords: Cholecystectomy; laparoscopy; pacemaker.

\section{Introduction}

Surgical procedures change as years go by and traditional surgical approaches are being replaced by minimally invasive techniques or endoscopic methods. The laparoscopic cholecystectomy (LC) procedure, which was first performed by Philippe Mouret in 1987, has become a common procedure in our country as it has in the world 
and also has become the gold standard in the treatment of symptomatic gallstone disease and other benign gallbladder diseases. ${ }^{[1,2]}$ Some conditions that initially constituted unequivocal contraindications for LC have gradually decreased by the utilization of novel developments in minimally invasive surgical methods. ${ }^{[3,4]}$ The indications of permanent pacemakers (PPM) and implantable cardioverter/ defibrillators (ICD) in clinical practice have gradually expanded and become a significant part of invasive cardiology. ${ }^{[5]}$ As a result, encountering such patients in noncardiac surgery is increasing in our frequency. Our hospital is a center where cardiac patients are diagnosed and treated on an intensive basis. The study at hand presents the results of patients with cardiovascular implantable electronic device (CIED) who had LC because of symptomatic gallstone disease.

\section{Materials and Methods}

\section{The Patients}

The medical records of patients who had LC at Kartal Koşuyolu Higher Specialty Training and Research Hospital's Gastroenterology Clinic between January 2012 and December 2016 were retrospectively evaluated. The study was made according to Helsinki Declaration. The files of the patients with CIED were analyzed. The data on the age, sex, existence of comorbidity, anticoagulant and antiaggregant agent administration, previous history of abdominal surgery, body mass index (BMI), American Society of Anesthesiologists (ASA) score, surgical indication, laboratory results, left ventricular ejection fraction (LVEF), type of CIED, indication of CIED implantation, perioperative CIED management, perioperative surgical and cardiac complications were recorded from the file records. Patient's duration of surgery, amount of blood loss, duration of postoperative hospitalization, and morbidity-mortality within the first 30 days were ascertained as well. An informed consent form was obtained from each patient for surgical intervention prior to surgery. All the patients were evaluated by cardiologists and anesthetists in the preoperative period and their evaluations on perioperative CIED management were obtained.

\section{Pacemaker Management}

Pacemakers were put on the asynchronous mode in the preoperative period or magnets were placed on the skin over the pacemakers in the operation room (OR) for ICD and pacemaker-dependent patients. The electrocautery plates were attached to the right ankles of such patients during the surgery. Bipolar electrocautery was preferably used. Short and intermittent touches were performed and the lowest possible amplitude was utilized by the electrocautery device when using monopolarelectrocautery. Continuous electrocardiography (ECG) and arterial pressure monitorization were achieved during the surgery. All the patients were closely followed-up in the surgical intensive care unit for about an hour after they were woken up. Patients with hemodynamic stability were transferred to the in-patient department. The pacemaker modes of the patients, which were changed before the surgery, were changed back to the old mode in the in-patient clinic following the surgery.

\section{Surgical Method}

The surgical procedures were performed under general anesthesia and in the reverse Trendelenburg position. The pneumoperitoneum was created by the Veress needle technique through a mini-incision done just below the umbilicus. The intraabdominal pressure was maintained at the $12 \mathrm{~mm}-\mathrm{Hg}$ level during the laparoscopy. The LC was performed according to the standard American technique. Two $10 \mathrm{~mm}$ trocars and two $5 \mathrm{~mm}$ trocars and reusable laparoscopy equipment were used. The $10 \mathrm{~mm}$ trocar in the gallbladder epigastric area was removed from its place. Only the fascia on the place of the trocar beneath the umbilicus was closed up with absorbable suture material. Skin incisions on other trocar areas were closed up with non-absorbable suture material.

\section{Statistical Analysis}

Statistical Package for the Social Sciences (SPSS 21 Inc., Chicago, IL, USA) computer software was used for bio-statistical analyses. When the data were presented as mean values their standard deviation values were given, when they were presented as median values their minimummaximum values were also stated.

\section{Results}

A total of 467 patients received LC at Kartal Koşuyolu Higher Specialty Training and Research Hospital's Gastroenterology Clinic between January 2012 and December 2016. Eight (0.017\%) patients had CIED. While 1 (12.5\%) of these patients was male, 7 (87.5\%) were female and the mean age of the patients was 53.2 (28-82 years). The mean BMI of the patients was $28.5 \mathrm{~kg} / \mathrm{m}^{2}(\min 22.9-\max 33.9)$. 
Five patients (62.5\%) were on aspirin, while $1(12.5 \%)$ patient was on oral warfarin treatment. Two (25\%) patients had no history of antiaggregant and anticoagulant agent use. When the LC indications of the patients were evaluated, it was seen that $7(87.5 \%)$ patients received LC because of symptomatic cholelithiasis while $1(12.5 \%)$ patient received LC because of acute cholecystitis. Table 1 summarizes the demographic and clinical characteristics of the patients. Table 2 presents the laboratory results of the patients. The surgical procedures were laparoscopically completed by the utilization of 4 ports as was defined previously in all the patients. The mean duration of surgery was 45.6 mins ( $\min 30-\max 60$ mins) and the intra-operational blood loss was not at a measurable level. The indication of pacemaker implantation was atrioven- tricular (AV) block in $5(62.5 \%)$ patients, hypertrophic cardiomyopathy (HCM) in $1(12.5 \%)$ patient, and dilated cardiomyopathy (DCM) in $2(25 \%)$ patients. While the pacemaker type for five patients was dual-chamber ratemodulated pacing (DDDR), it was ICD for 3 patients. When the CIED management was evaluated, it was observed that the pacemakers of $6(75 \%)$ patients were put on the preoperative asynchronous mode. Magnets were placed on the pacemakers of two (25\%) patients intraoperatively. No postoperative cardiac or surgical complications were seen in none of the patients following surgery. The mean duration of postoperative hospitalization of the patients was 1.75 days (min 1-max 2 days) (Table 3). Neither morbidity nor mortality was seen in any of the patients within the first postoperative 30 days.

\section{Table 1. The demographic and clinical characteristics of the patients}

\begin{tabular}{lccccccccc}
$\begin{array}{l}\text { Patient } \\
\text { No }\end{array}$ & Age & Gender & $\begin{array}{c}\text { Additional } \\
\text { comorbidity }\end{array}$ & $\begin{array}{c}\text { Abdominal } \\
\text { surgery } \\
\text { history }\end{array}$ & $\begin{array}{c}\text { Antiagregan- } \\
\text { anticoagulant } \\
\text { drug use }\end{array}$ & $\begin{array}{c}\text { Indication } \\
\text { of } \\
\text { surgery }\end{array}$ & $\begin{array}{c}\text { BMI } \\
\left(\mathbf{k g} / \mathbf{m}^{2}\right)\end{array}$ & $\begin{array}{c}\text { ASA } \\
\text { score }\end{array}$ & $\begin{array}{c}\text { LVEF } \\
(\%)\end{array}$ \\
\hline 1 & 28 & Female & MR & C/S & None & Cholelithiasis & 32.5 & 2 & 60 \\
2 & 46 & Male & CAD, HT & None & Aspirin & Cholelithiasis & 25.7 & 3 & 65 \\
3 & 38 & Female & CAD (MI history) & C/S & Aspirin & Cholelithiasis & 26.5 & 3 & 65 \\
4 & 59 & Female & CAD & None & Aspirin & Cholelithiasis & 30.8 & 3 & 55 \\
5 & 82 & Female & CAD, HT, DM & None & Aspirin & Acute & 22.9 & 3 & 60 \\
& & & & cholecystitis & & & \\
6 & 64 & Female & CAD, HT, DM, CHF & None & Aspirin & Cholelithiasis & 27.3 & 3 & 40 \\
7 & 42 & Female & AVR & None & Warfarin & Cholelithiasis & 33.2 & 2 & 60 \\
8 & 67 & Female & HT, CHF & None & None & Cholelithiasis & 29.2 & 3 & 40 \\
\hline
\end{tabular}

BMI: Body mass index; ASA: American Society of Anesthesiologists; LVEF: Left ventricular ejection fraction; MR: Mitral regurgitation; CAD: Coronary artery disease; HT: Hypertension; MI: Myocardial infarction; DM: Diabetes mellitus; CHF: Congestive heart failure; AVR: Aort valv replasman; C/S: Sectio.

\section{Table 2. The laboratory results of the patients}

\begin{tabular}{lccccccc}
\hline Patient No & WBC $\left(10^{3} / \mu \mathrm{L}\right)$ & HCT $(\%)$ & PLT $\left(10^{3} / \mu L\right)$ & CRE $(\mathbf{m g} / \mathbf{d L})$ & ALT $(\mathrm{U} / \mathrm{L})$ & BIL $(\mathbf{m g} / \mathbf{d L})$ & INR \\
\hline 1 & 5900 & 33.5 & 277 & 0.51 & 20 & 0.8 & 1.04 \\
2 & 9600 & 43.9 & 203 & 0.91 & 18 & 0.84 & 1.12 \\
3 & 8800 & 41.5 & 220 & 0.65 & 27 & 0.4 & 1.06 \\
4 & 8500 & 42.2 & 313 & 0.58 & 20 & 0.7 & 1.05 \\
5 & 10700 & 31.8 & 175 & 1.09 & 82 & 1.1 & 1.04 \\
6 & 9900 & 41.6 & 207 & 0.65 & 21 & 0.6 & 1 \\
7 & 5400 & 33.9 & 290 & 0.6 & 22 & 0.3 & 0.99 \\
8 & 7600 & 39.8 & 235 & 1.3 & 20 & 0.11 & 0.99 \\
\hline
\end{tabular}

WBC: White blood cells; HCT: Hematocrit; PLT: Platelet; CRE: Creatinin; ALT: Alanine aminotransferase; BIL: Total bilirubin; INR: International Normalized Ratio. 
Table 3. The CIED types of the patients, intraoperative and postoperative results

\begin{tabular}{lcccccccc}
$\begin{array}{l}\text { Patient } \\
\text { No }\end{array}$ & $\begin{array}{c}\text { Indication } \\
\text { of CIED }\end{array}$ & $\begin{array}{c}\text { CIED } \\
\text { type }\end{array}$ & $\begin{array}{c}\text { CIED } \\
\text { management }\end{array}$ & $\begin{array}{c}\text { Duration } \\
\text { of surgery } \\
\text { (min) }\end{array}$ & $\begin{array}{c}\text { Intraoperative } \\
\text { blood } \\
\text { loss }(\mathbf{m l})\end{array}$ & $\begin{array}{c}\text { Postoperative } \\
\text { complication }\end{array}$ & $\begin{array}{c}\text { Hospital } \\
\text { stay } \\
\text { (day) }\end{array}$ & $\begin{array}{c}\text { Postoperative } \\
\text { 30-day } \\
\text { mortality }\end{array}$ \\
\hline 1 & AV Block & DDDR & Asynchronous & 30 & NA & None & 1 & None \\
2 & AV Block & ICD & Asynchronous & 45 & NA & None & 2 & None \\
3 & HCM & ICD & Asynchronous & 60 & NA & None & 1 & None \\
4 & AV Block & DDDR & Magnet & 40 & NA & None & 2 & None \\
5 & AV Block & DDDR & Asynchronous & 60 & NA & None & 2 & None \\
6 & DCM & DDDR & Magnet & 40 & NA & None & 2 & None \\
7 & AV Block & DDDR & Asynchronous & 40 & NA & None & 2 & None \\
8 & DCM & ICD & Asynchronous & 50 & NA & None & 2 & None \\
\hline
\end{tabular}

CIED: Cardiovascular implantable electronic device; AV: Atrioventricular; HCM: Hypertrophic cardiomyopathy; DCM: Dilated cardiomyopathy; DDDR: Dual-chamber rate-modulated pacing; ICD: Implantable cardioverter-defibrillator; NA: None available.

\section{Discussion}

Today LC is the gold standard for the treatment of symptomatic gallstone disease and it is a procedure frequently performed at general surgery clinics. The greatest advantages of LC can be summarized as alleviated postoperative pain, rapid return to postoperative normal physical activity, and better cosmetic results. ${ }^{[1,2]}$ Pacemakers are used for various indications notably for bradyarrhythmia cases. ICDs, on the other hand, were started to be used in the treatment of tachyarrhytmia cases in the clinical practice and became a significant part of cardiac invasive procedures. ${ }^{[5]}$ The presence of CIED has important effects on the preoperative, intraoperative, and postoperative patient management. Patients with CIED can be taken into surgery if appropriate measures are taken. ${ }^{[6]}$ An underlying cardiac disease which may include arrhythmic conditions like sinoatrial node dysfunction, AV block, atrial fibrillation (AF), and ventricular tachycardia (VT) is always present in patients with CIED. The Heart Rhythm Society (HRS) and the ASA have jointly issued an expert consensus statementin July 2011, which was also approved by the American College of Cardiology (ACC) and the American Heart Association (AHA), in order to assist clinicians in the perioperative evaluation and management of patients with CIED. An active communication between the surgical team and the clinician is the basis of any successful perioperative management and should happen prior to the surgical procedure in an outpatient with CIED. ${ }^{[7]}$ CIED management is affected by such factors as whether the pacemaker has unipolar or bipolar ends, whether the electrocautery device is bipolar or unipolar, the distance between the elec- trocautery device and the pacemaker, the severity of the patient's dependence on the pacemaker, and whether the surgical procedure to be performed is elective or emergency. ICD devices should be reprogrammed immediately or sometime after the surgery. ${ }^{[8]}$

According to the perioperative management recommendations in patients with PPM and ICD presented in the 2014 guidelines of the European Society of Cardiology (ESC) and the European Society of Anaesthesiology (ESA), monopolarelectrocautery use constitutes a great risk for patients as electrical excitation coming from the electrocautery device might block or reprogram pacemakers during surgical procedures. Such problems can be avoided or minimized by utilizing bipolar electrocautery devices and accurately positioning the grounding plate for the electrical circuit. Keeping the electrocautery device away from the pacemaker, releasing only short bursts, and using the lowest amplitude possible might also decrease statics. The PPM should be programmed to asynchronous or undetected modes in pacemaker-dependent patients. This can be done in the easiest way in the ORs by placing a magnet on the skin over the PPM. Patients with unreliable underlying rhythms should have their pacemakers questioned after the surgery in order to enable the appropriate programming and detection-speed limits. ICD function can be intervened during non-cardiac surgical procedures as a result of the electrical current produced by the electrocautery device. ICDs should be turned off during the surgery and should be synchronized before the patients' discharge. The defibrillating function of an ICD can temporarily be deactivated by placing a magnet on the skin 
over the ICD. An external defibrillator should be provided while the device is being deactivated. ${ }^{[9]}$

There is only a limited number of studies in literature on the safe performance of LC in patients with CIED. The results of our study, which was also conducted with a limited number of patients, revealed that LC that is a minimally invasive procedure can safely be performed in patients with CIED. The limitations of our study include its being retrospective with a limited patient population, the absence of comparisons with other patient groups with LC, and its being single-centered.

\section{Conclusion}

LC is one of the most commonly performed surgical procedures in general surgery clinics. The possibility that surgeons may perform surgical procedures on patients with CIED for various causes increases day by day. All the centers in our country might not have experienced clinics and clinicians that are experienced in CIED procedures. This situation is especially significant in unplanned emergency surgical procedures. We, consequently, believe that LC can safely be performed in patients with CIEDs through accurate perioperative management by experienced centers.

\section{Disclosures}

Ethichs Committee Approval: Retrospective study.

Financial Disclosure: The authors declared that this study has received no financial support.

Peer-review: Externally peer-reviewed.

Conflict of Interest: None declared.

\section{References}

1. Cuschieri A, Dubois F, Mouiel J, Mouret P, Becker H, Buess G, et al. The European experience with laparoscopic cholecystectomy. Am J Surg 1991;161:385-7. [CrossRef]

2. Alper A, Emre A, Bilge 0 , Gençosmanoğlu R, Acarlı K, Arıoğul O. Laparoscopic cholecystectomy: The results of 200 cases.
Turk J Surg 1994;10:248-53.

3. Soper NJ, Stockmann PT, Dunnegan DL, Ashley SW. Laparoscopic cholecystectomy. The new 'gold standard'? Arch Surg 1992;127:917-21. [CrossRef]

4. [No authors listed] National Institutes of Health Consensus Development Conference Statement on Gallstones and Laparoscopic Cholecystectomy. Am J Surg 1993;165:390-8.

5. Mirowski M, Reid PR, Mower MM, Watkins L, Gott VL, Schauble JF, et al. Termination of malignant ventricular arrhythmias with an implanted automatic defibrillator in human beings. $\mathrm{N}$ Engl J Med 1980;303:322-4. [CrossRef]

6. Healey JS, Merchant R, Simpson C, Tang T, Beardsall M, Tung S, et al; Canadian Cardiovascular Society; Canadian Anesthesiologists' Society; Canadian Heart Rhythm Society. Canadian Cardiovascular Society/Canadian Anesthesiologists' Society/Canadian Heart Rhythm Society joint position statement on the perioperative management of patients with implanted pacemakers, defibrillators, and neurostimulating devices. Can J Cardiol 2012;28:141-51. [CrossRef]

7. Crossley GH, Poole JE, Rozner MA, Asirvatham SJ, Cheng A, Chung MK, et al. The Heart Rhythm Society (HRS)/American Society of Anesthesiologists (ASA) Expert Consensus Statement on the perioperative management of patients with implantable defibrillators, pacemakers and arrhythmia monitors: facilities and patient management this document was developed as a joint project with the American Society of Anesthesiologists (ASA), and in collaboration with the American Heart Association (AHA), and the Society of Thoracic Surgeons (STS). Heart Rhythm 2011;8:1114-54. [CrossRef]

8. Eagle KA, Berger PB, Calkins H, Chaitman BR, Ewy GA, Fleischmann KE, et al; American College of Cardiology; American Heart Association. ACC/AHA guideline update for perioperative cardiovascular evaluation for noncardiac surgery-executive summary: a report of the American College of Cardiology/American Heart Association Task Force on Practice Guidelines (Committee to Update the 1996 Guidelines on Perioperative Cardiovascular Evaluation for Noncardiac Surgery). J Am Coll Cardiol 2002;39:542-53. [CrossRef]

9. Kristensen SD, Knuuti J, Saraste A, Anker S, Bøtker HE, Hert SD, et al. 2014 ESC/ESA Guidelines on non-cardiac surgery: cardiovascular assessment and management: The Joint Task Force on non-cardiac surgery: cardiovascular assessment and management of the European Society of Cardiology (ESC) and the European Society of Anaesthesiology (ESA). Eur Heart J 2014;35:2383-431. [CrossRef] 